\title{
Could Proterozoic positive carbon isotope excursions be tracking intense methane fluxes to the atmosphere? An analogue- based hypothesis
}

CAdeAu P ${ }^{1}$, Ader M', JeZequel D ${ }^{1}$, Foullland E², Le FlOCH' E ${ }^{2}$, SARAZIN G' ${ }^{1}$ BERNARD C ${ }^{3}$, LEBOULANGER C

- Université de Paris, Institut de Physique du Globe de Paris, CNRS, Paris, France

2 MARBEC, Univ Montpellier, IRD, CNRS, Ifremer, Sète, France.

Muséum National d'Histoire Naturelle, UMR MCAM, Paris, France

The Proterozoic era presents distinct extreme positive carbon isotope excurions (CIEs) usually interpreted as a global perturbation of $\mathrm{C}$-cycle due to an increased organic carbon burial. This interpretation bears strong consequences on our understanding of the ocean and atmosphere oxygenation. However, these positive CIEs do not correspond very well with other tracers of oxygenation and present a large variability of amplitude, timing and duration that is not as compatible with a unique global perturbation of the $\mathrm{C}$ cycle as currently assumed.

We propose here that some of the most positive CIEs may also reflect regional desequilibria between the inorganic carbon dissolved in the water and the atmospheric $\mathrm{CO}_{2}$, induced by an active methanogenesis associated to a substantial methane outgassing.

This conceptual scenario is supported by observations performed in a modern ecosystem: the Dziani Dzaha crater lake (Mayotte, Indian Ocean). The results we obtained from this ecosystem allow us not only to identify several other analogies with some Proterozoic environments but also to demonstrate that its positive CIE is indeed due to active methanogenesis and methane escape to the atmosphere

If this new scenario was to be more firmly established for a few Proterozoic positive CIEs, it would open new perspectives to better understand the interactions between the $\mathrm{C}-$, S- and $\mathrm{O}$-cycles and the climate regulation during the Proterozoic. 